\title{
Effects and Interactions of agile Principles in the Process of Mechatronic System Development: Building a basic Un- derstanding for adaptive Process Design
}

\author{
Albert Albers $^{1}$, Jonas Heimicke ${ }^{1}$, Sebastian Trost ${ }^{1}$ \\ ${ }^{1}$ IPEK - Institute of Product Engineering, Karlsruhe Institute of Technology (KIT) \\ \{Albert.Albers, Jonas.Heimicke, Sebastian.Trost\}@kit.edu
}

\begin{abstract}
Background: In order to be as reactive as possible to changing conditions caused by uncertainties in the markets and therfore in the product development environment, companies are increasingly implementing agile approaches in mechatronic systems development. These mostly originate from the software branch and are based on the culture of this branch, thus creating various challenges in the culture of mechatronic system development. The approach of ASD - Agile Systems Design is based on 9 core principles for agile mechatronic system development. The operationalization of these principles leads to a situation- and demandoriented degree of agility in the development process. However, the effects of these principles are only implicitly known, so that the successful implementation of agility through ASD - Agile Systems Design is based on the experiences of the developers. Aim: For the purposeful implementation of the agile core principles, the effects they cause in the development process are to be understood and their interdependency examined. This should create the basis for the sustainable implementation of cultural and technical agility in the processes of mechatronic system development. Method: In order to understand the effects of the core principles, 12 successful product development projects in which agile was developed were retrospectively examined the data basis is the project documentation. The resulting understanding was sharpened in two stages with 23 experts from research and practice and the effects of the principles were made explicit. Results: The result of the research work are nine effect clusters, which are caused by the core principles of ASD, as well as the presentation of the interdependencies of the principles. Conclusions: By explicating the effects, a generic framework was created which on the one hand supports the implementation of cultural agility and on the other hand enables the development of processes, methods and tools which promote the sustainable implementation of technical agility based on the needs in mechatronic system development. In addition, desired effects can now be induced prospectively in a targeted manner, since they are known and explicit.
\end{abstract}

Keywords: product development, uncertainty, process improvement, ASD - Agile Systems Design, Mechatronic System Development 


\section{Introduction}

Western markets have increasingly developed towards consumers paying less for owning a product and more for using a product. This has resulted in new cash flow strategies, an increased emphasis on services such as maintenance and repair and a change in consumer behaviour. (Mont, 2002) This means that companies no longer sell physical commodities on the market, but rather product-service systems that represent a combination of tangible goods and services (Gausemeier \& Plass, 2014, p. 160). In addition to advantages such as the possibility of expanding the product portfolio and business plans, new challenges arise for companies. For example, traditional corporate structures need to be changed and customer integration in product development processes needs to be strengthened. (Mont, 2002) Product service systems are a consequence of the trade-off between mass production and customization and realize the concept of mass customization (Da Silveira, Borenstein, \& Fogliatto, 2001). This leads inevitably to the fact that a large number of new and different objectives have to be satisfied by the value offered on the market, the number of interacting socio-technical systems is rising and products are increasingly being developed across different domains, which means that product development has to be considered in terms of the system of systems thought in order to cope with the interactions between systems (Keating et al., 2003). Since in particular interfaces in the later operational context of a technical system are unknown (usually the developers do not know with which systems a developed system will interact), the development of a product service system is a complex venture (Albers, Mandel, Yan, \& Behrendt, 2018). This makes it difficult to plan product development processes and to comply with the plan (Boehm \& Turner, 2003; Snowden \& Boone, 2007), which is why agile approaches are also increasingly becoming part of the processes of the companies in the area of physical product development (Schmidt et al., 2019). According to Albers, Basedow et al., services and business models are an integral part of mechatronic system development today, which is why these components will be consistently considered in the further course of this article (Albers, Basedow et al. 2020). These approaches follow agile principles, which are fundamental guidelines for successful operation in the sense of agile development practices, but mostly originate from the software development industry (Fowler \& Highsmith, 2001). However, there are also various approaches based on agile principles for mechatronic system development that are better suited to the conditions here than software development principles (Albers, Heimicke, Spadinger et al., 2019; Mussgnug, Boes, \& Meboldt, 2015). In order to be able to apply the principles in projects of mechatronic systems development in the sense of the system-of-systems concept, the effects that are intended to be achieved by following the principles in development projects are identified in this paper and the principles are analysed with regard to their interdependence. This creates an understanding of agile principles in mechatronic system development, which is the basis for a successful prospective application of the agile principles in future projects.

\section{State of the Art}

\subsection{Product Development and the Path to Agility}

The development of Product-Service-Systems can be understood as a continuous interaction of three systems. The operation system contains all resources (developer, knowledge, infrastructure, etc.) necessary for a successful development. It creates and concretizes the system of objectives, which contains all objectives that are to be fulfilled by a product to be developed, their justification, interactions and requirements associated with the objectives. Based on the system of objectives, the operation system develops the system of objects, which summarizes all results generated in a development (sketches, prototypes, the final product service system). The system 
of objectives and the system of objects interact exclusively via the operation system and are iteratively extended in continuous sequences of synthesis and analysis as shown in Figure 1. left (Albers \& Lohmeyer, 2012). This process occurs in a specific product engineering context, which Gericke et al. (2013) breaks down into additional subgroups based on the levels of resolution (Figure 1. right) proposed by Hales and Gooch (2004, p. 21). These are in turn assigned factors that define the context on the respective level and can lead to a development environment of varying complexity depending on the constellation of these factors (Gericke et al., 2013).

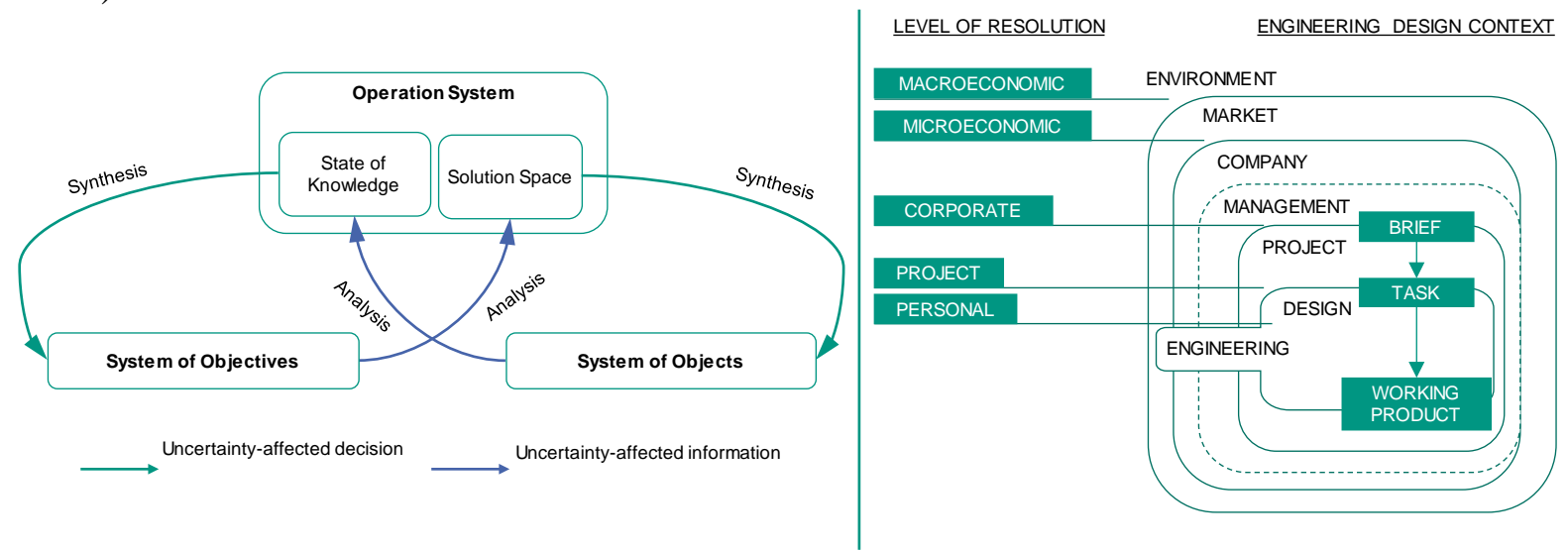

Figure 1. Left: extended System Triple (Albers \& Lohmeyer, 2012); Right: structuring the context of product engineering (Hales \& Gooch, 2004)

Agility in product development refers to the ability of an operation system to respond to changing contextual conditions and to adapt the sequence of synthesis and analysis activities (to make changes in the project plan (Conforto, Amaral, da Silva, Di Felippo, \& Kamikawachi, 2016)), in order to achieve the greatest possible value for customers, users, and providers through the product (Albers, Heimicke, Müller, \& Spadinger, 2019). On the other hand, there are the plandriven approaches to product development, which demand a definition of product requirements at the beginning of a project (Petersen \& Wohlin, 2010) and, based on this, the planning of long time horizons. They are particularly suitable in stable environments with controllable context conditions. (Boehm \& Turner, 2003) Although the character of the respective project is decisive for the suitability of any approach (agile or plan-driven) (Heimicke, Freire, Breitschuh, \& Albers, 2019; Snowden \& Boone, 2007), many companies are currently striving to increasingly introduce agile approaches into their processes (Schmidt, Paetzold, \& Weiss, 2018). However, when introducing these new approaches, companies are faced with new challenges that they must deal with specifically (Dikert, Paasivaara, \& Lassenius, 2016; Goevert, Heimicke, Lindemann, \& Albers, 2019; Heimicke, Niever et al., 2019; Schmidt et al., 2019). For example, organizational change in order to implement agility more effectively is characterized by numerous challenges, like a wrong mindset, resistance to change or cultural issues (Gandomani \& Nafchi, 2016) and often requires a change in the entire organizational culture (Dikert et al., 2016). Agile product development is not only based on the use of individual tools or practices, but on a holistic mindset (Misra, Kumar, \& Kumar, 2010). Agile transformation can therefore also be considered an organizational mutation because the scope of the required changes includes all organizational behaviors as well as the roles and responsibilities of the people involved (Gandomani \& Nafchi, 2016). Karvonen et al. note that for a holistic agile transformation of a company, it must focus on the unique and sophisticated interaction of operational, strategic or cultural aspects (Karvonen, Sharp, \& Barroca, 2018). Diebold et al. consider agility from two perspectives: technical agility and cultural agility, where methods and practices are part of technical agility and values and principles are part of cultural agility. They also emphasize that in the context of the "Agile Transition" the consideration of cultural agility is often neglected and 
instead methods, such as Scrum, are only considered from a technical perspective. However, sustainable agility can only be achieved through a symbiosis of these two perspectives. (Diebold, Küpper, \& Zehler, 2015)

\subsection{ASD - Agile Systems Design}

An approach based on principles for the agile development of mechatronic systems and thus providing organizational units with processes, methods and tools derived from these values is the approach of ASD - Agile Systems Design (Albers, Heimicke, Spadinger et al., 2019). Based on these core principles, a company- and context-specific adaptation of development processes and the provision of methods to implement a suitable level of agility in the development context for the respective project is performed. The nine principles of ASD are not always equally important and they do not provide a recipe for the right agile approach. Rather, they serve as guidelines for developers in different organizational units to ensure that the respective operations in the development process comply with these guidelines. The core principles of ASD Agile Systems Design are (Albers, Heimicke, Spadinger et al., 2019):

1. The developer is the center of product development

2. Each product development process is unique and individual

3. Agile, situation- and demand-oriented combination of structuring and flexible elements

4. Each process element can be located in the system triple and each activity is based on the fundamental operators analysis and synthesis

5. All activities in product engineering are to be understood as a problem-solving process

6. Each product is developed on the basis of references

7. Product profiles, invention and business model form the necessary components of the innovation process

8. Early and continuous validation serves the purpose of continuous comparison between the problem and its solution

9. For a situation- and demand-oriented support in every development project, methods and processes must be scalable, fractal and adaptable

For the purposeful operationalisation of the core principles Albers et al. (2020) use the subcategories to describe the context of product development identified by Gericke et al. (2013). However, Albers et al. (2020) do not use these categories to identify context factors. Rather, they have assigned over 200 factors to these categories based on the literature, which influence the agile capabilities of these organizational units at the respective organizational levels. For example: Group: Company $\rightarrow$ Subgroup: Stakeholder $\rightarrow$ Factors: Agile oriented condition management; Involvement of internal and external stakeholders; Integration of customers, suppliers and users; Regulated cross-company collaboration

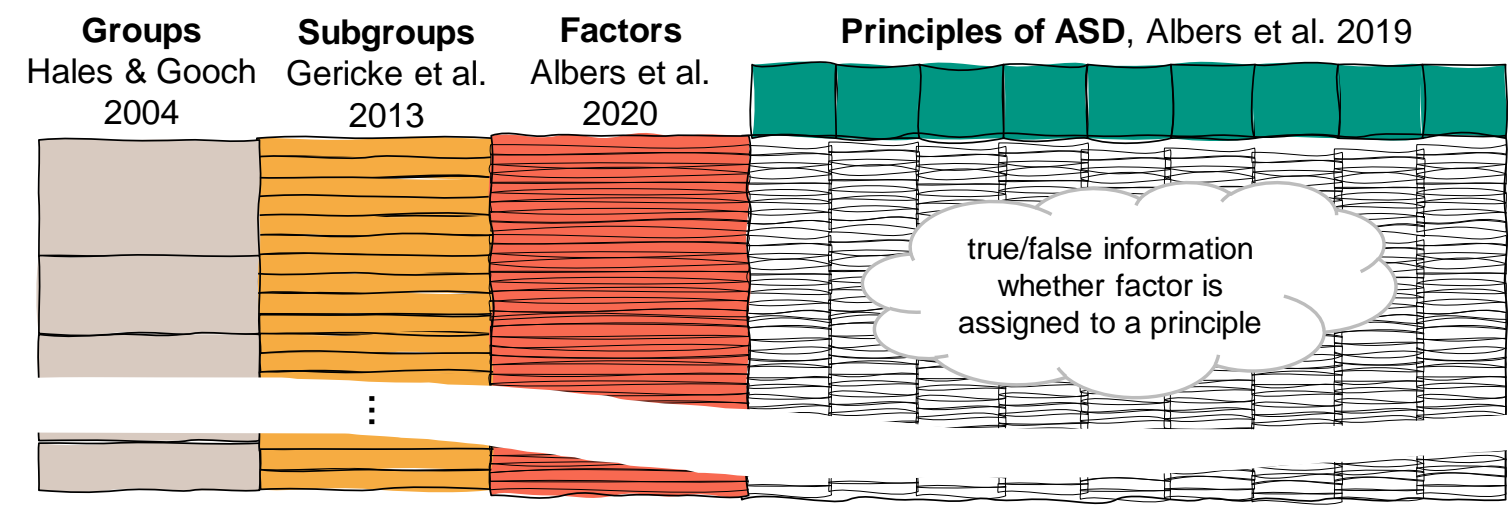

Figure 2. Matrix for linking the ASD principles with the factors influencing agility (Albers et al. 2020) 
In addition, they have assigned the factors to those ASD core principles which, when operationalized, have a positive influence on the respective factor and influence it in the sense of agility. Thus, there is a direct correlation between the core principles of ASD, the factors that have an influence on agility in the company and the specific subgroups and main groups (Figure 2.) (Albers et al., 2020) If, for example, a process author in a company is encouraged by his management to implement agile approaches, he can now define the focus of the agile approach by weighting the subcategories. In addition, the influencing factors associated with the highest rated subcategories can be weighted in the second step. As a result, the user receives weighted core principles of ASD - Agile Systems Design, which in combination with the factors reflect a profile of methods or processes that must be served by an agile approach in this specific case. This can be adapted to the specific needs in order to provide the best possible support for the developers. (Albers et al., 2020)

\section{Research profile}

In various agile projects, methods and processes of ASD - Agile Systems Design have already been successfully applied (Albers, Heimicke et al., 2018). Nevertheless, the selection and use of these methods and processes was mostly based on the experience of participating developers in the field of project management. In order to be able to use the agile principles of ASD Agile Systems Design in a more purposeful way in the future and to adapt methods and processes for the operationalization of these principles to the respective needs of an organization and the development context at hand, a deeper understanding of the effects that are achieved by implementing the ASD principles in the development process is required. In addition, interactions between the principles must be examined in more detail in order to identify synergies and conflicts that result in the area of agile product development with ASD. To achieve this goal, the following research questions are answered in this paper.

1. Which effects in the development process are caused by following agile principles of mechatronic system development?

2. What is the connection between the agile principles of mechatronic system development?

The effects of the principles were determined in five stages. First, the documented development processes (project plans and real project histories) of 12 development projects were analysed qualitatively (Albers, Heimicke et al., 2018) and recurring elements such as activities or methods (Albers, Rapp et al., 2019) were identified (stage 1). Based on this, in the course of this research, the occurring effects of those elements were reconstructed and assigned to the ASD core principles (stage 2). The collected effects were expanded and formulated in an expert workshop with more than 10 researchers from the field of product development (stage 3). In a further workshop with 23 experts from practice and research, challenges were collected and compared with the effects (stage 4). The resulting final formulation of the effects was again validated by the same committee as in the third stage (stage 5).

For the connection between the agile principles we have identified a quantitative criterion. This is based on the connectivity analysis carried out by Albers, Heimicke et al. (2020) on the basis of the factors identified in the literature that were linked to the agile principles (a complete list of the more than 200 factors can be found in (Albers, Heimicke et al., 2020)). Based on this matrix we interlinked certain principles more closely if they positively influence a larger number of the same factors. 


\section{Effects of following core principles of ASD - Agile Systems Design}

Assuming that product developers follow the nine core principles of ASD - Agile Systems Design when developing mechatronic systems, certain effects can be expected in the development process that support successful product development. The effects, which were explained by the four-step procedure described in Chapter 3, were formulated with the aim of being able to be applied to different development contexts. The derived effects of each principle are listed below:

Effects of the 1st principle: The developer is the center of product development:

- Development in cross-functional teams including employee-centered leadership

- Provision of intuitive methods, processes and tools tailored to the situation, competences and requirements of the developers

- Establishment of framework conditions for the promotion of creativity (open spaces, infrastructures, ...)

- Use of mechanisms for the demand-oriented provision of knowledge and resources

- Establishment of structures to increase the motivation and commitment of employees

- Enabling autonomous work through empowerment (authorization, transfer of responsibility)

- $\quad$ Targeted sensitisation of employees to lifelong learning (methods/programmes)

Effects of the 2nd principle: Each product development process is unique and individual:

- Enable project-specific planning of the development process (no cooking recipe)

- Creation of free space to realize a good enough planning (plan only as far as you can)

- Mechanisms for using existing process knowledge in project planning

- Use of Mechanisms for communication and transparent provision of individual project objectives

- Project-specific provision of resources and composition of core teams

- Use of mechanisms to identify and reduce redundant work steps

- Use of mechanisms for the project-specific classification of the project in terms of development risk

Effects of the 3rd principle: Agile, situation- and demand-oriented combination of structuring and flexible elements:

- Creation of superordinate structures (e.g. milestones) and definition of generic deliverables at the beginning of the project

- Allow and define iterations or sequences on different process levels (project, phases, activities, methods)

- Use of mechanisms to identify the necessity (uncertainties, complexity, lack of planning stability) of maturity level specific iterations

- Use of mechanisms for the identification of agile and sequential development strands

- Regularly questioning the appropriateness of the respective approach used in development

- $\quad$ Not starting the project with a list of requirements

- Establishing structures to enable the development team to be highly responsive

Effects of the 4th principle: Each process element can be located in the system triple and each activity is based on the fundamental operators analysis and synthesis:

- Separation of objectives and objects in the development process

- Enabling the selection of methods and processes depending on the level of maturity of the system of objectives and objects

- Creation of framework conditions that encourage/support the targeted implementation of iterations 
- Enabling and inducing a co-evolution of objectives and objects through mutual synthesis and analysis activities

- Use of mechanisms for targeted growth of the knowledge base and continuous narrowing / detailing of the solution space

- Creating a high level of situational awareness to check the usefulness of activities and targeted work

- Raising the awareness of the development team to carry out validation activities early and continuously, validating not only objects but also objectives

Effects of the 5th principle: All activities in product engineering are to be understood as a problem-solving process:

- Dividing activities into structured sub-activities

- Introduction of a uniform structuring of problem-solving processes

- Creation of a high level of situation and problem awareness through a uniform understanding of the concept of the problem

- Enable a situation-adequate detailing of problem solving activities (granularity via fractality)

- Enable intuitive localization in the development process by combining development and problem solving activities

- Facilitating access to development methods appropriate to the situation and needs

- Establishment of a regular review and adjustment of the problem solving team

Effects of the 6th principle: Each product is developed on the basis of references:

- Use of Mechanisms for a well-founded assessment of development risks by means of product knowledge

- Use of mechanisms to increase innovation potential through conscious identification of reference system elements

- Enabling the utilization of the creativity potential of developers through targeted stimulation in the process (e.g. TRIZ)

- Creation of easy and targeted access to existing product and associated process knowledge (databases, experts, lead users, etc.)

- Creating a conscious handling of new development shares to support the design and, if necessary, adjustment of the project plan

- Use of existing product knowledge to further develop the validation and/or production system

- Creation of framework conditions that allow employees to adopt new perspectives/expand their horizons

Effects of the 7th principle: Product profiles, invention and business model form the necessary components of the innovation process:

- Orientation of product development towards the fulfilment of customer, user and provider benefits (product profile)

- Creation of structures to integrate relevant stakeholders and their objectives into the development process

- Enable change of perspective for systematic consideration of product benefits (e.g. via customer engineer)

- Targeted use of creativity methods to develop new solutions

- Targeted use of construction methods for the technical implementation of new solutions to meet requirements

- Continuous development of the product profile based on the system of objects

- Early and targeted alignment of the solution space for invention and business model through the product profile 
Effects of the 8th principle: Early and continuous validation serves the purpose of continuous comparison between the problem and its solution:

- Validation of the system-in-development in the context of higher-level systems in which it is embedded, the system environment and the system user, using specific maneuvers

- Continuous monitoring of the fulfilment of customer, user and provider benefits throughout the development process, for example by integrating them into q-gates

- Ensuring early validation of the development direction, minimizing risks and avoiding late changes

- Conscious selection of the specification type of the System-in-Development (virtual, physical, mixed) in validation

- Ensure early gain of knowledge through continuous review of prioritized requirements / assumptions (verification)

- Use of the PGE model for "efficient" validation through systematic principle variation (PV), embodiment variation (EV) and carryover variation (CV) on the existing validation system

- Validation system is continuously "co-developed" with the product

Effects of the 9th principle: For a situation- and demand-oriented support in every development project, methods and processes must be scalable, fractal and adaptable:

- Enable scaling of mindsets, methods and processes depending on the size / scope of the development project at the beginning of the project

- Enable dynamic scaling of mindsets, methods and processes as needed during the course of the project (e.g. allowing an unplanned iteration)

- Promotion of a simple adaptation of mindsets, methods and processes to different development contexts

- Create mechanisms for intuitive scaling and easy achievement of the required granularity of mindsets, methods and processes (fractal characteristics)

- Elimination of interface problems across team / department /company boundaries

- Ensuring an appropriate exchange of information and clear understanding of roles, responsibilities and areas of responsibility

- Targeted scaling and use of methods, ways of thinking and processes analogous to the considered system level in the context of System of Systems

By explicating the effects, the understanding of the individual core principles of ASD and their effects was deepened. For the implementation of ASD, the effects are useful because it is now possible for method and process developers to select specific practices or methods to trigger the intended effects within the process. Accordingly, the effects are elements of the requirement profile for the process solution to be developed using Agile Systems Design. Furthermore, by explicating the effects, a basis was created to make the degree of implementation of individual core principles measurable in the future.

\section{Interactions of the core principles of ASD - Agile Systems Design}

The explicitly described effects of the individual core principles of ASD have already been used to derive relations between the ASD core principles and agility-influencing factors, which made it possible to create the network matrix shown in Figure 2. According to this, a factor that has an influence on the agility of an operation system is assigned to a core ASD principle if both elements serve at least one common effect. By counting the number of factors assigned to each individual ASD core principle, the general relevance of a principle can be estimated. If a principle has been assigned a particularly large number of factors, it has an influence on the agile capabilities of the operation system in broad application cases, and thus such a principle can generally be assigned a higher relevance than a principle to which a significantly smaller 
number of factors has been assigned. Therefore, especially principle 1 (The developer is the center of product development) is relevant in many use cases (see Figure 3.). The network matrix also shows the interaction between the individual ASD principles. Thus, whenever a single factor has been assigned to several principles, there is a correlation between the principles. In Figure 4. the interaction of all nine core principles of the ASD is shown, where the size of a circle is proportional to the sum of the implications of a principle and thus describes the interactivity of the principle. The strength of the connecting line between two principles is proportional to the common implications that the connected principles have. Principles that have a particularly high degree of interactivity implicitly contain information of other principles and influence the degree of fulfilment of the other principle by their own fulfilment.

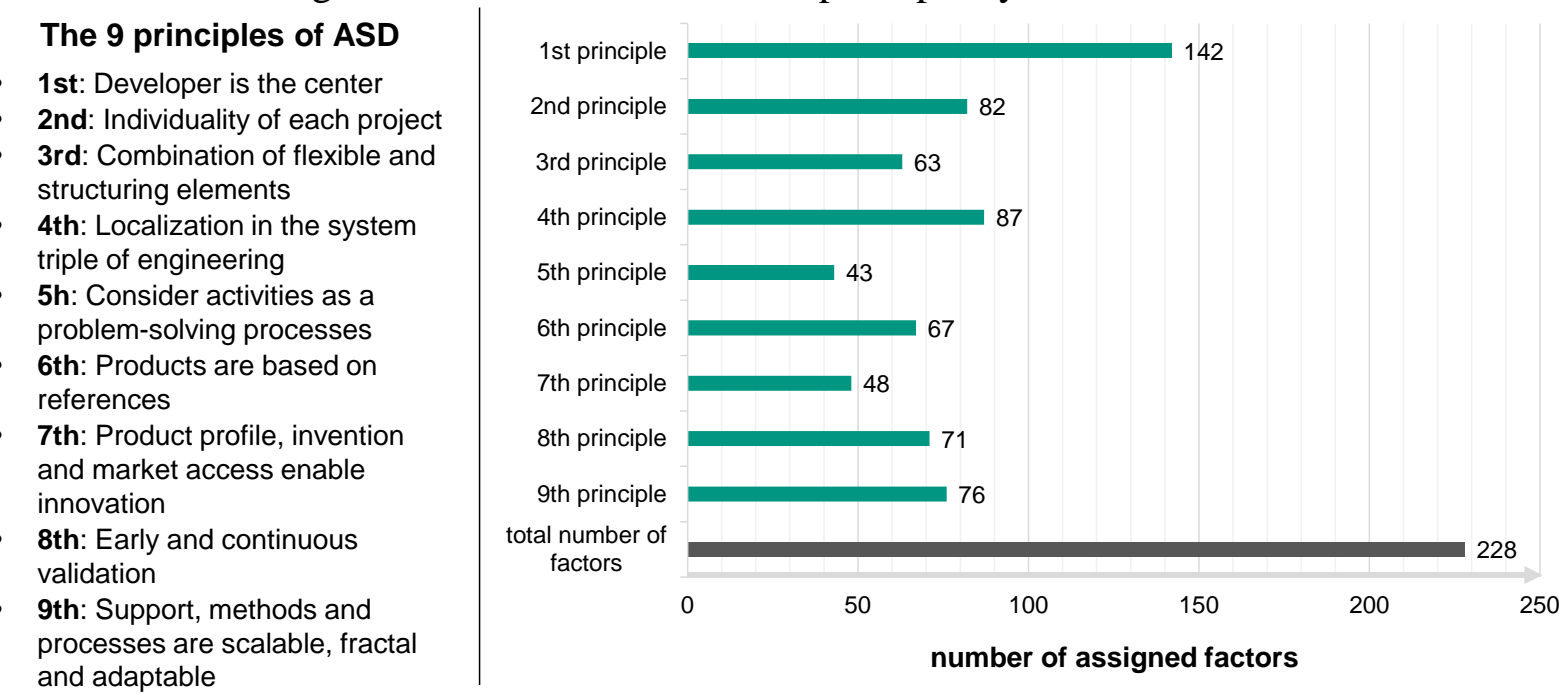

Figure 3. Number of factors assigned to the respective ASD core principles that influence the agile capabilities of an operation system

This insight in the figure below can provide method and process developers with a better understanding of the overall scope of ASD. In addition, principles with high interactivity can now be specifically influenced in order to benefit from synergy effects.

The 9 principles of ASD

- 1st: Developer is the center

- 2nd: Individuality of each project

- 3rd: Combination of flexible and structuring elements

- 4th: Localization in the system triple of engineering

- 5h: Consider activities as a problem-solving processes

- 6th: Products are based on references

- 7th: Product profile, invention and market access enable innovation

- 8th: Early and continuous validation

- 9th: Support, methods and processes are scalable, fractal and adaptable

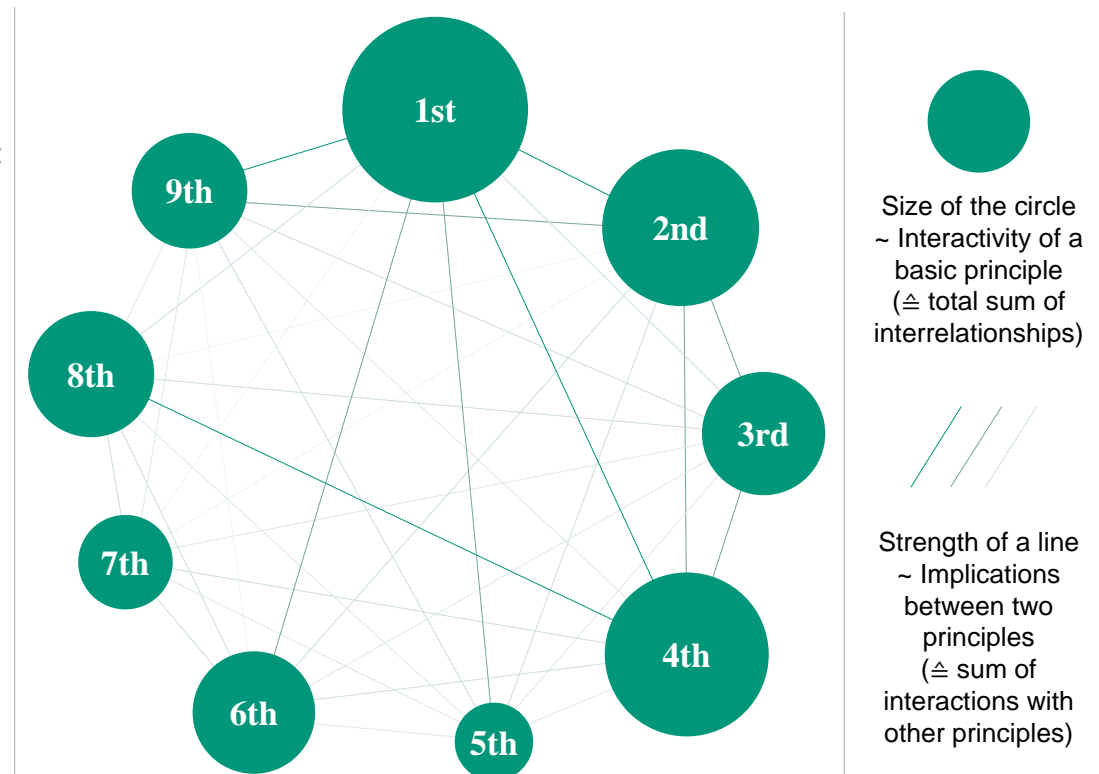

Figure 4. Implications and interactions of the ASD core principles 
In the illustrations given here, it should be noted that the factors received have no weighting at all, but each factor influencing agility was considered equally important. For specific situations and demands in the development process, however, it cannot be assumed that individual factors have the same weight. For this reason, the statements on the representations made here are of a generic nature and should not be used for individual case considerations.

\section{Discussion and Conclusion}

Agile approaches, which have been repeatedly proven to be successful in software development processes, are increasingly used in mechatronic systems development. However, the most commonly used agile techniques such as Scrum or eXtreme Programming are based on values and principles that originate from the culture of the software industry. For the successful application and sustainable perpetuation of agile practices in mechatronic systems development, agile methods, processes and tools must follow the culture of the manufacturing industry. The present research aims to create a basis for the cultural and technical introduction of agility that is appropriate for mechatronic system development and reflects the culture that exists here.

The contribution of this article is an investigation of existing core principles of ASD - Agile Systems Design for agile mechatronic system development regarding their effects in the development process. These effects are to be induced by the prospective and targeted use of suitable methods, processes and tools in development projects. Thus, in this paper a clear understanding of the effects of the 9 core principles of ASD - Agile Systems Design was established. For this purpose, 12 real development projects were retrospectively analysed and the effects identified from the documentation were deepened and explicated in expert workshops. In addition, based on a network analysis from Albers et al. (2020), the connection between the core principles was analysed. It was found that the principle the developer is the centre of product development is the principle with the greatest influence on the success of a development project on the one hand, and on the other hand it contains the greatest implications for the other 8 principles. This means that in most cases this principle should be implemented together with the other principles. However, all principles are connected with each other; but the strength of the connection varies. Based on the findings of the present work, in which the understanding of cultural agility in mechatronic system development was deepened, suitable methods, processes and tools for the sustainable introduction of technical agility and the operationalization of the nine core principles of ASD - Agile Systems Design in development processes of mechatronic system development can now be (further) developed.

\section{References}

Albers, A., Basedow, G. N., Heimicke, J., Marthaler, F., Spadinger, M., Rapp, S. (2020). Developing a common understanding of business models from the product development perspective. Proceedings of 30th CIRP Design, in print.

Albers, A., Heimicke, J., Müller, J., \& Spadinger, M. (2019). Agility and its Features in Mechatronic System Development: A Systematic Literature Review. Proceedings of 30th ISPIM Innovation Conference.

Albers, A., Heimicke, J., Spadinger, M., Reiss, N., Breitschuh, J., Richter, T., . . Marthaler, F. (2019). A systematic approach to situation-adequate mechatronic system development by ASD - Agile Systems Design. Procedia CIRP, 84, 1015-1022. https://doi.org/10.1016/j.procir.2019.03.312

Albers, A., Heimicke, J., Trost, S., \& Spadinger, M. (2020). Alignment of the change to agile through method-supported evaluation of agile principles in physical product development. Proceedings of 30th CIRP Design Conference, in print. 
Albers, A., Heimicke, J., Walter, B., Basedow, G. N., Reiß, N., Heitger, N., . . . Bursac, N. (2018). Product Profiles: Modelling customer benefits as a foundation to bring inventions to innovations. Procedia CIRP, 70(1), 253-258. https://doi.org/10.1016/j.procir.2018.02.044

Albers, A., \& Lohmeyer, Q. (2012). Advanced systems engineering - towards a model-based and human-centered methodology. Proceedings of TMCE 2012, 407-416.

Albers, A., Mandel, C., Yan, S., \& Behrendt, M. (2018). System of systems approach for the description and characterization of validation environments. In DS92: Proceedings of the DESIGN 2018 15th International Design Conference. https://doi.org/10.21278/idc.2018.0460

Albers, A., Rapp, S., Peglow, N., Stuermlinger, T., Heimicke, J., Wattenberg, F., ～\& Wessels, H. (2019). Variations as Activity Patterns: A Basis for Project Planning in PGE - Product Generation Engineering. Procedia CIRP, pp. 966-972. https://doi.org/10.1016/j.procir.2019.04.314

Boehm, B., \& Turner, R. (2003). Using risk to balance agile and plan- driven methods. Computer, 36(6), 57-66. https://doi.org/10.1109/MC.2003.1204376

Conforto, E. C., Amaral, D. C., da Silva, S. L., Di Felippo, A., \& Kamikawachi, D. S. L. (2016). The agility construct on project management theory. International Journal of Project Management, 34(4), 660-674. https://doi.org/10.1016/j.ijproman.2016.01.007

Da Silveira, G., Borenstein, D., \& Fogliatto, F. S. (2001). Mass customization: Literature review and research directions. International Journal of Production Economics, 72(1), 1-13. https://doi.org/10.1016/S0925-5273(00)00079-7

Diebold, P., Küpper, S., \& Zehler, T. (2015). Nachhaltige agile transition: Symbiose von technischer und kultureller Agilität. Projektmanagement Und Vorgehensmodelle 2015.

Dikert, K., Paasivaara, M., \& Lassenius, C. (2016). Challenges and success factors for largescale agile transformations: A systematic literature review. Journal of Systems and Software, 119, 87-108.

Fowler, M., \& Highsmith, J. (2001). The Agile Manifesto. Softw. Dev. 9, 28-35.

Gandomani, T. J., \& Nafchi, M. Z. (2016). Agile transition and adoption human-related challenges and issues: A Grounded Theory approach. Computers in Human Behavior, 62, 257-266.

Gausemeier, J., \& Plass, C. (2014). Zukunftsorientierte Unternehmensgestaltung: Strategien, Geschäftsprozesse und IT-Systeme für die Produktion von morgen (2., überarb. Aufl.). München: Hanser. https://doi.org/10.3139/9783446438422

Gericke, K., Meißner, M., \& Paetzold, K. (2013). Understanding the context of product development. Proceedings of the 19th International Conference on Engineering Design (ICED13) Design for Harmonies, 75(3).

Goevert, K., Heimicke, J., Lindemann, U., \& Albers, A. (2019). Interview Study on the Agile Development of Mechatronic Systems. In Design Society (Chair), Proceedings of the 22nd International Conference on Engineering Design (ICED19), Delft, The Netherlands. https://doi.org/10.1017/dsi.2019.235

Hales, C., \& Gooch, S. (2004). Managing Engineering Design (Second Edition). London: Springer. https://doi.org/10.1007/978-0-85729-394-7

Heimicke, J., Freire, G., Breitschuh, J., \& Albers, A. (2019). Determining degrees of complexity of different development situations within business processes in engineering sector. 41st R\&D Management Conference 2019 “The Innovation Challenge: Bridging Research, Industry and Society”.

Heimicke, J., Niever, M., Zimmermann, V., Klippert, M., Marthaler, F., Albers, A. (2019) Comparison of Existing Agile Approaches in the Context of Mechatronic System Development: Potentials and Limits in Implementation. In Proceedings of the 22nd 
International Conference on Engineering Design (ICED19), Delft, The Netherlands, 58 August 2019. https://doi.org/10.1017/dsi.2019.226

Karvonen, T., Sharp, H., \& Barroca, L. (2018). Enterprise agility: Why is transformation so hard? In International Conference on Agile Software Development (pp. 131-145).

Keating, C., Rogers, R., Unal, R., Dryer, D., Sousa-Poza, A., Safford, R., .. . Rabadi, G. (2003). System of Systems Engineering. Engineering Management Journal, 15(3), 3645. https://doi.org/10.1080/10429247.2003.11415214

Misra, S. C., Kumar, V., \& Kumar, U. (2010). Identifying some critical changes required in adopting agile practices in traditional software development projects. International Journal of Quality \& Reliability Management.

Mont, O.K. (2002). Clarifying the concept of product-service system. Journal of Cleaner Production, 10(3), 237-245. https://doi.org/10.1016/S0959-6526(01)00039-7

Mussgnug, M., Boes, S., \& Meboldt, M. (2015). Principles guiding teams in new product development projects. Proceedings of EPDE 2015, Loughborough, UK, 56-61.

Petersen, K., \& Wohlin, C. (2010). The effect of moving from a plan-driven to an incremental software development approach with agile practices. Empirical Software Engineering, 15(6), 654-693. https://doi.org/10.1007/s10664-010-9136-6

Schmidt, T. S., Atzberger, A., Gerling, C., Schrof, J., Weiss, S., \& Paetzold, K. (2019). Agile Development of Physical Products: An Empirical Study about Potentials, Transition and Applicability: Report, University of the German Federal Armed.

Schmidt, T. S., Paetzold, K., \& Weiss, S. (2018). Agile Entwicklung physischer Produkte. VDIStatusreport 09/2018.

Snowden, D. J., \& Boone, M. E. (2007). A Leader's Framework for Decision Making. Harvard Business Review, 85(11), 68-77. 